\title{
Direct imaging search for substellar companions around neutron stars
}

\author{
B. Posselt ${ }^{1,2}$, R. Neuhäuser ${ }^{1}$ and F. Haberl ${ }^{2}$ \\ ${ }^{1}$ Astrophysikalisches Institut und Universitätssternwarte, \\ FSU Jena, Schillergässchen 3-5, 07745 Jena, Germany \\ ${ }^{2}$ Max-Planck-Institut fuer extraterrestrische Physik, \\ Giessenbachstrasse, Garching, Germany \\ email: posselt@mpe.mpg.de
}

\begin{abstract}
Currently two planetary systems around pulsars are known - discovered by the pulse timing technique, an indirect method. These planets were a surprise and gave rise to diverse planet formation scenarios, some of them very different to the common planet formation models around solar type stars and thus physically very interesting. Furthermore, neutron star planets are not only interesting themselves but also to study properties of the poorly understood neutron stars. After a summary about the current state of pulsar planets and the theoretical formation models, we present our own direct-imaging search for thermal emission of neutron star planets using the VLT. The project sample includes the fascinating radio-quiet isolated neutron stars, which are some of the closest and probably youngest neutron stars we know. Companions around them can only be found by direct imaging. Detecting planets around neutron stars by direct imaging differs significantly from using this technique for other, e.g. solar type, stars. As great advantage there is no need to reject the starlight of the primary.
\end{abstract}

Keywords. Stars: neutron, Infrared.

\section{Introduction}

The first earth-mass planets discovered have been neutron star planets. Meanwhile four planets are known around the old millisecond(ms)-pulsar PSR 1257+12 ( Wolszczan \& Frail (1992), Konacki \& Wolszczan (2003), Wolszczan (2005)) . They have up to four earth masses and are very close to the primary - $0.2 \mathrm{AU}$ to around $3 \mathrm{AU}$. Interestingly, for three of them nearly circular orbits are reported (Konacki \& Wolszczan (2003)). Another planetary system around a neutron star is the binary system of the ms-pulsar PSR 1620+10 and its white dwarf companion (Sigurdsson (2003), Sigurdsson \& Thorsett (2004)). Actually, a 2.5 Jupiter mass object is orbiting the white dwarf at 23 AU.

These unxepected dicoveries rised several questions about the formation of these planets. Did they formed before the supernova and how they did survive this event ? If they formed after the supernova, how did they form ? In the case of PSR $1257+12$ it is extremely unlikely that all four planets are captured from other stars, while this is the likely explanation of PSR $1620+10$ where probabaly an exchange of the white dwarf companion took place (Sigurdsson \& Thorsett (2004)). There are several models for planet formation after the supernova or survival, well described by Podsiadlowski (1995) and references therein. If the planets around a neutron star survived the supernova, an initial orbital separation larger than 4 AU (to survive the giant stage) and asymmetric supernovae are needed. The nearly circular orbits of three planets make this survival model unlikely for PSR $1257+12$. For formation after the supernova, the supernova fallback model, the circumbinary disk model and the disrupted companion model are currently 
discussed (e.g. Podsiadlowski (1995)). The first formed disks have different properties. e.g. in masses, sizes and composition. The following planet formation could differ from those around solar-like stars. Thus, the study of neutron star planetary systems in comparison to those around solar-like stars can be very fruitful for the understanding of planet formation in gerneral. For PSR $1257+12$ the circumbinary disk model and the disrupted companion model are currently favoured (Podsiadlowski (1995)).

But substellar companions of neutron stars are not only interesting for planet formation theory. They provide also insights into the supernova event and maybe the progenitor star(s). Furthermore, the study of low-mass companions around neutron stars, their masses and orbital characteristics, can give masses of the neutron stars. Together with radius determinations these neutron star masses constrain the neutron stars (degenerate) equation-of-state. Currently no radius and mass of a neutron star are known at the same time and the equation of state of ultradense matter in the Universe is one of the big questions in astrophysics. While masses of neutron stars can be derived for binary systems (e.g. Jonker et al. (2003)), the best radius estimation comes from the radio-quiet X-ray thermal neutron stars, which are isolated without stellar companions (e.g. Trümper et al. (2004)).

\section{How to find substellar companions around neutron stars?}

By radio pulse arrival time variations, one can detect only relatively close companions (like in radial velocity planet searches among normal solar-like stars), so that the question of wide companions remains open. Furthermore only radio pulsars with very stable pulses, like ms-pulsars can be investigated. Radio-quiet neutron stars are not covered. As the neutron stars are very faint (fainter than 25 mag in the optical), the commonly used radial velocity method or the search for astrometric wobbling should preferably be done in Xrays. Unfortunately the spectral and spatial resolution in X-ray astronomy is currently not enough for these methods. Furthermore the neutron stars have active region, pulsar beams and even larger companions than the primary, which also rule out the transit method as good choice. Substellar companions of all possible separations can be detected only by direct imaging, especially in the near infrared if they are young (They are several mags fainter in the optical.). The companions are brighter than the neutron stars in the NIR, even though lower in mass, because they are larger in size and still contracting when young. Direct detection of very close and faint substellar companions is currently possible only around neutron stars.

\subsection{Direct Imaging Search}

\subsubsection{Sample selection}

The magnitudes of substellar objects (brown dwarfs and giant planets) can be predicted following the non-gray theory of substellar objects by Burrows et al. (1997), who have computed IR magnitudes of substellar objects depending on age and mass etc. At 100 Myrs and $300 \mathrm{pc}$, a companion at the Deuterium burning mass limit ( 13 $\left.\mathrm{M}_{\mathrm{jup}}\right)$ would have an H-band magnitude of $22 \mathrm{mag}$ (Burrows et al. (1997)). In case that possible companions to the neutron stars formed before the supernova, their age is the sum of the age of the progenitor star and the time elapsed since the neutron stars was born as such (in the supernova). The former is negligible compared to the latter. We have therefore chosen to select all neutron stars younger than 100 Myrs and closer than 300 pc.

Within 300 pc there are seven radio plusars younger than 100 Myrs, selected from the pulsar catalog of Taylor et al. (1993) in its latest online revision. To that sample, we 
added the seven radio-quiet neutron stars discovered recently as very bright and soft Xray sources with ROSAT. These neutron stars are also called X-ray thermal neutron stars, because they are thermal X-ray emitters, showing no non-thermal component, especially no radio emission; for reviews, see e.g. Neuhäuser \& Trümper (1999), and Haberl (2004). Their blackbody-like spectra results in the best radius estimation currently avaiable (Trümper et al. (2004)), making them especially interesting for the determination of the equation of state of neutron stars. The X-ray brightest dim thermal neutron star (RX J1856.5-3754) was identified as a V=25.7 mag star using both the HST (Walter \& Matthews (1997)) and the NTT (Neuhäuser et al. (1998)). For two dim thermal neutron stars only upper limits $(R>23 \mathrm{mag}, V>24 \mathrm{mag})$ are avaiable, but the limits presumably exclude everything except an isolated neutron star (Haberl (2004) and references therein). The remaining four have magnitudes up to $B=27.2 \mathrm{mag}$ (Motch et al. (2005), Haberl (2004), Haberl et al. (2004)). These deep optical observation exclude all companions down to substellar masses, hence these neutron stars are isolated.

The ages of the radio pulsars in our sample are known to be $\leqslant 100$ Myrs from the spindown time. The additional radio-quiet neutron stars found as bright and soft ROSAT sources are most probably also young neutron stars, indicated by large proper motion and high magnetic fields. They are probably several Myrs old, with cooling (but still hot) surfaces which emit the X-ray emission. For all seven radio-quiet X-ray thermal neutron stars, it appears unlikely that they are old with X-ray emission due to accretion from the interstellar medium, because their X-ray emission is constant, and also for statistical reasons (Neuhäuser \& Trümper (1999)). Three radio-quiet X-ray thermal neutron stars have a known proper motion, which is so high, that it implies too low Bondi-Hoyle accretion efficiencies to explain these stars as old neutron stars re-heated by accretion from the ISM. Thus, 100 Myrs can be savely used as an upper age limit for all 14 neutron stars.

Most of 14 neutron stars are at high galactic latitude and for all fields, we know that the absorbing column density is quite low. For the radio pulsars, distance estimates are available from the dispersion measure (Taylor et al. (1993)). For the radio-quiet Xray thermal neutron stars, a direct optical detection of the neutron star itself and the measurement of its parallax is available only in one case, namely RX J1856.5-3754 (the brightest in X-ray and, hence, probably also the brightest in the optical, V=25.7 mag), for which Walter \& Lattimer (2002) determined 117 pc. The other X-ray thermal neutron stars appear to be very similar. The measured column densities from the X-ray spectra are smaller than the galactic values (towards the respective direction), which means that these neutron stars are also quite nearby. One can assume the same order for the distance as for RX J1856.5-3754, i.e. roughly 100 pc.

\subsubsection{First epoch observations}

Of the 14 young nearby neutron stars twelve were observed in $H$ or $K$-band using the VLT with the instrument ISAAC, the Keck telescope (NIRCSPEC) or the $2.2 \mathrm{~m}$ telescope of the University of Hawaii (QUIRC). We considered possible orbital separation up to $5000 \mathrm{AU}$, taking into account recently found wide substellar companions at around $4000 \mathrm{AU}$ and possible increase of orbits due to the mass loss of the primary due to the supernova. Using deep optical observation (e.g. down to 26 mag in $B$ or $V$ ) avaiable for some of the neutron stars we can derive colors and roughly estimate spectral types of faint NIR-objects. Comparing these color to the know ones for M-dwarfs (e.g. Kenyon \& Hartmann (1995)) we can already exclude many probable stars from further considerations. In nine out of the twelve observed fields there are several candidates up to 5000 AU. As an example the field of RX J0720.4-3125 is shown in Fig. 1. The deep 


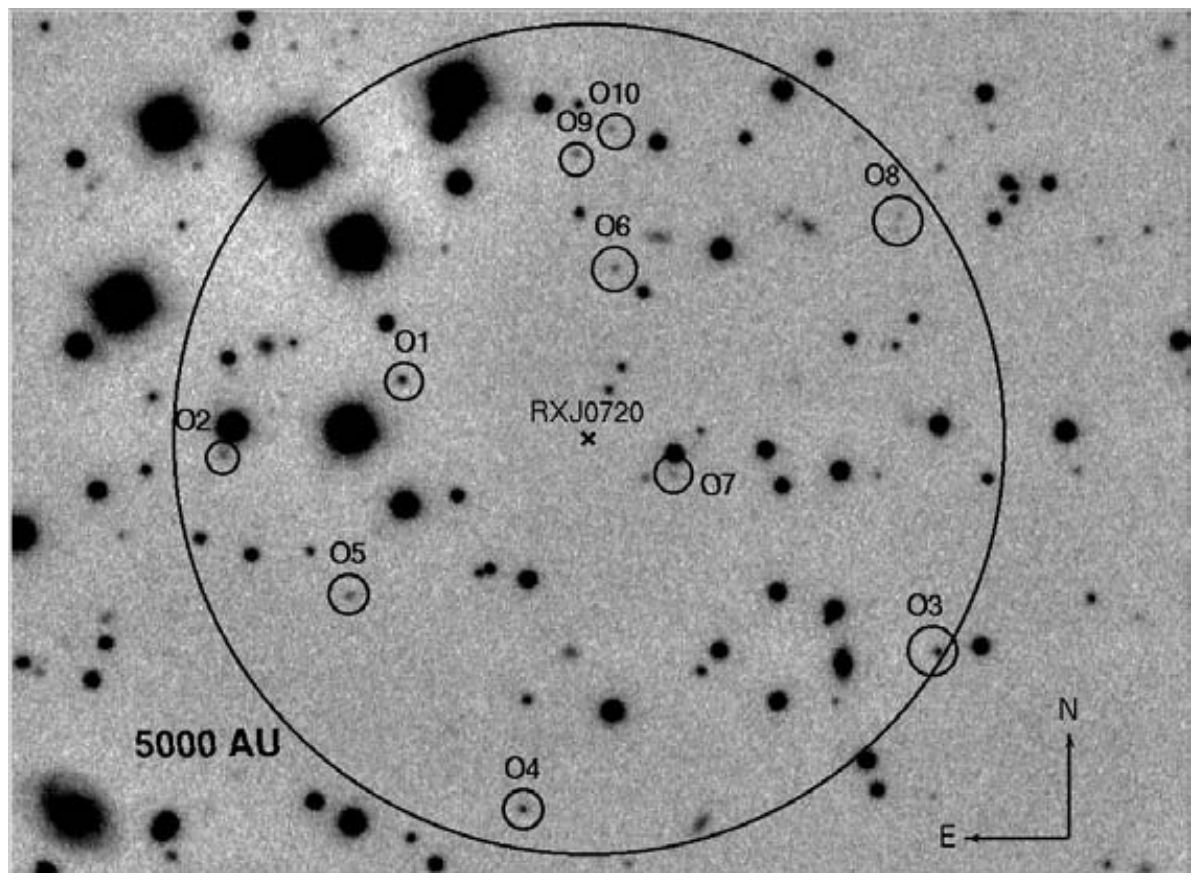

Figure 1. This 1st epoch ISAAC H-band image of RX J0720.4-3125 shows interesting substellar candidates considered for 2nd epoch observation (box size: $75 \operatorname{arcsec} \times 55 \operatorname{arcsec}$ ). The neutron star in the center (RA: 07:20:24.92, DEC: -31:25:50.90) is surrounded by a circle of $5000 \mathrm{AU}$ radius, which was searched for possible substellar companion candidates having at least a SNR of three. The indicated sources O1 to O10 are faint enough to be substellar companion candidates. The magnitudes range from $\mathrm{H}=20.9 \mathrm{mag}(\mathrm{O} 1)$ to $\mathrm{H}=22.3 \mathrm{mag}(\mathrm{O} 10)$. O5 to $\mathrm{O} 10$ are faint enough $(\mathrm{H} \gtrsim 21.5 \mathrm{mag})$ to be even planet candidates following the estimations by Burrows et al. (1997). The B-H color of $\mathrm{O} 4$ excludes a star earlier than $\mathrm{M} 6 \mathrm{~V}$ as counterpart. For all other candidate objects the FORS1 B-band limit (28 mag) results in B-H colors larger than 5.5, excluding stars earlier than $\mathrm{M} 2 \mathrm{~V}$ as counterparts.

optical observations are VLT FORS1 B-band observations by Motch et al. (2005) of the same year.

\subsubsection{Outlook/ Future observations}

The faint substellar companions candidates found around the nine neutron stars can not be confirmed using only their JHK-colors. From JHK, one could indeed simultaneously determine reddening and spectral type (i.e. temperature). However, even if the color is consistent with a substellar companion (of spectral type $\mathrm{L}$ or $\mathrm{T}$ ) and if the background possibility would be very unlikely (from galactic latitude, angular separation, and faintness), the object could still be an unrelated reddened background object. Thus, proper motion confirmation is necessary. The known proper motion for the investigated neutron stars range from around $40 \mathrm{mas} / \mathrm{yr}$ to $300 \mathrm{mas} / \mathrm{yr}$. For the fastest neutron stars the second epoch observations will be carried out soon. Together with JHK-colors and - if bright enough - even spectra the nature of the found substellar companions can be clearified.

Future extremely large telescopes like the planned $30 \mathrm{~m}$ Telescope (TMT webpage (2005)), the $50 \mathrm{~m}$ EURO50 (EURO50 webpage (2005)) or the $100 \mathrm{~m}$ OWL Telescope (ESO's OWL webpage (2005)) would increase the search possibilities significantly. Even the lowest-mass gas giant planets would be seen within our search criteria of young and 
closeby neutron stars. Alternatively, one can also expand the search to older objects (e.g. $1 \mathrm{Gyr}$ ) or more far away neutron stars. According to the recent pulsar catalogue (ATNF Pulsar Database (2005)) one could cover around 40 young pulsars (up to $1.1 \mathrm{kpc}$ ), 80 (up to $1.9 \mathrm{kpc}$ ) or 120 young pulsars (up to $3.8 \mathrm{kpc}$ ) by the use of $30 \mathrm{~m}, 50 \mathrm{~m}$ or $100 \mathrm{~m}$ telescope respectively. Hopefully by the time of the first light of these telescopes also more than the seven radio-quiet X-ray thermal neutron stars have been found, especially interesting to get constraints on the equation of state of ultadense matter in the universe.

\section{References}

The ATNF Pulsar Database September 2005, atnf.csino.au/research/pulsar/psrcat Burrows, A. et al. 1997, ApJ 491, 865

ESO OWL webpage 2005, www.eso.org/projects/owl

EURO50 webpage 2005, www.astro.lu.de/\% YEtorben/euro50/

Haberl, F. 2004, AdSpR 33, 638

Haberl, F. et al. 2004, A\&SA 424, 635

Jonker, P.G., Van der Klis, M., \& Groot, P.J. 2003, MNRAS 339, 663

Kenyon, S.J. \& Hartmann L. 1995, ApJS 101, 117

Konacki, M. \& Wolszczan, A. 2003, ApJ 591, 147

Motch, C. 2005, A\& A 429, 257

Neuhäuser, R. et al. 1998, Msngr 92, 27

Neuhäuser, R. \& Trümper, J. 1999, A $₫ A$ 143, 151

Podsiadlowski, P. 1995, ASP Conference Series, 72

Sigurdsson, S. et al. 2003, Science 301, 193

Sigurdsson, S. \& Thorsett, S.E. 2004, ASP Conference Series TBD

Taylor, J.H., Manchester, R.N., \& Lyne, A.G. 1993, ApJS 88, 529

Trümper, J. et al. 2004, Nucl. Phys. 132C, 560

TMT webpage 2005, tmt.ucolick.org

Walter, F.M. \& Matthews, L.D. 1997, Nature 389, 358

Walter, F.M. \& Lattimer, A.G. 2002, ApJ 576, 145

Wolszczan, A. \& Frail, D.A. 1992, Nature 355, 145

Wolszczan, A. 7. February 2005, www.science.psu.edu/alert/wolszczan2-2005.htm 

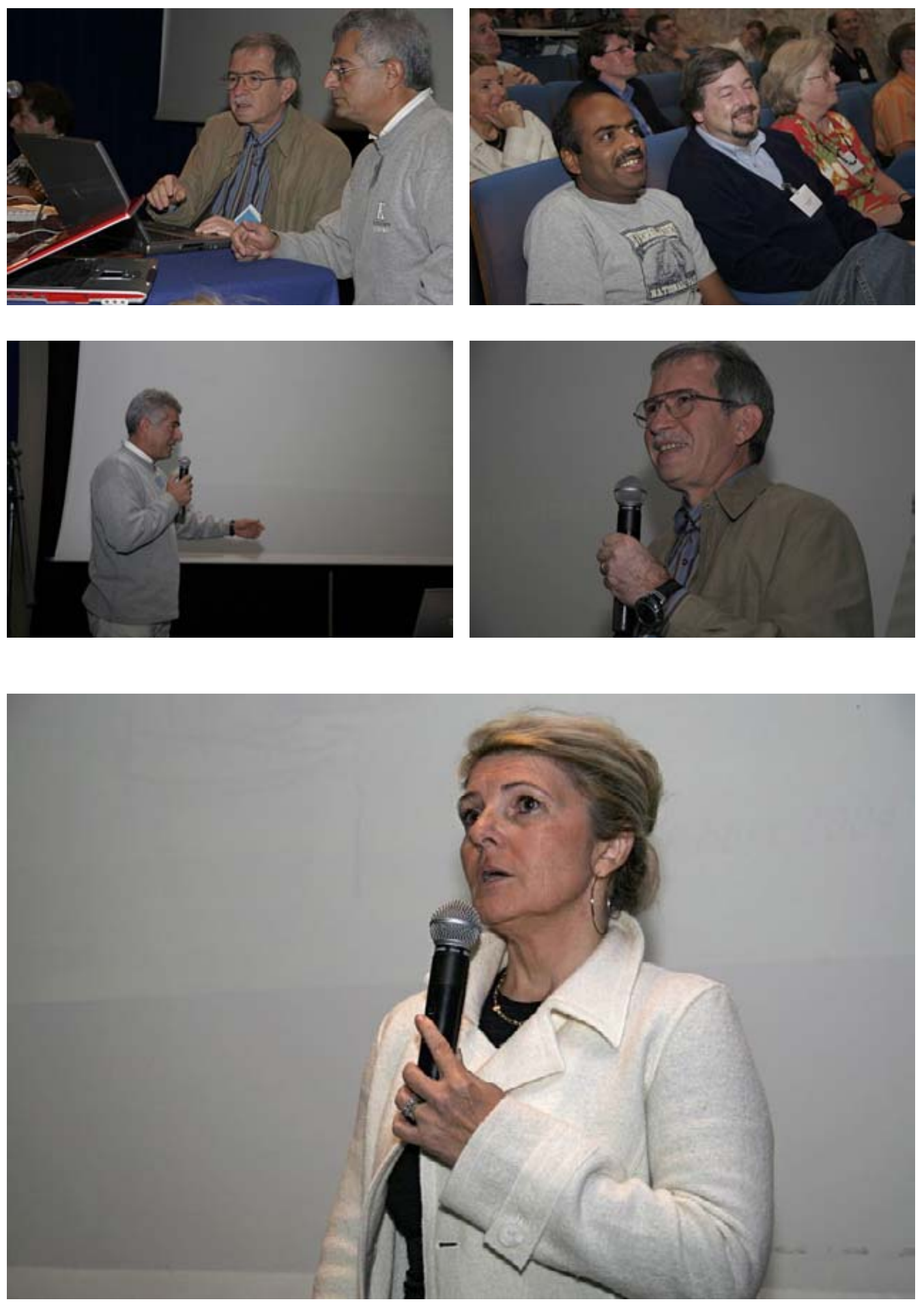

All photographs: Laurent Thareau [1.thareau@free.fr]. 